of complete health. Some months later two of this generation produced healthy litters, and the feeding experiments are being continued with the third generation. There was no disturbance of bowel action at any stage, in the direction of either constipation or diarrhoea. There was no hardening of the stools or pitting of the surface of the faeces, as noted by other observers in the case of animals fed with other bulky insoluble powders.

Three animals of the first generation were killed after seven months' feeding, during which time each had consumed an amount of trisilicate approximately equal to its own original body weight-equivalent to considerably more than a hundredweight for an average man. Two animals of the second generation were killed after four months' continuous trisilicate feeding. The intestinal tracts, their contents, and the various viscera were carefully examined, and sections were cut of the stomach, small intestine, colon, liver, pancreas, spleen, kidney, lung, thyroid, thymus, suprarenal, and heart muscle, but there was no evidence of morbid change in any part.

Many other post-mortem examinations were carried out on other species with similar results. In every case the trisilicate was found to be distributed evenly along the alimentary tract and mixed intimately with the contents. There was no evidence of sedimentation, the formation of concretions, or other localized accumulations in any area.

Other systematic feeding experiments were carried out on young adult white mice. The daily dose per animal was 0.23 gram (ignited weight), corresponding to about 10 grams per kilo, or 14 grams of the parent substance. The animals grew normally, and had every appearance of health (see Table VII), producing litters after a few months

TABLE VII.-Mice

[Daily dose of magnesium trisilicate $=0.3 \mathrm{gram}(0.23 \mathrm{gram}$ ignited weight)]

\begin{tabular}{l|c|c|c|c|c|c|c|c|c}
\hline Mouse & $\begin{array}{l}\text { Original } \\
\text { Weight }\end{array}$ & \multicolumn{2}{|c|}{ Monthly Average of Weekly Weighings } & $\begin{array}{c}\text { Final } \\
\text { Weight } \\
\text { Sept. }\end{array}$ & $\begin{array}{c}\text { Total } \\
\text { Gain }\end{array}$ \\
\cline { 3 - 8 } & April & May & June & July & Aug. & & \\
\hline 1 & 22 & 23 & 24 & 25 & 25 & 25 & 27 & 5 \\
2 & 24 & 24 & 25 & 25 & 27 & 30 & 31 & 7 \\
3 & 23 & 24 & 25 & 25 & 25 & 24 & 25 & 2 \\
4 & 22 & 21 & 20 & 21 & 19 & 21 & 23 & 1 \\
5 & 26 & 26 & 28 & 29 & 30 & 30 & 30 & 4 \\
6 & 17 & 19 & 21 & 21 & 20 & 21 & 20 & 3 \\
\hline
\end{tabular}

of feeding. At the end of five months of trisilicate feeding they were all killed, and naked-eye and histological examinations were performed exactly as in the case of rats. Again it was impossible to detect any morbid change in the digestive tract or viscera.

\section{Human Dosage}

Single doses varying from 1 drachm to 1 ounce of the parent powder were given to twelve volunteers without the production of any kind of symptom. Daily doses were then given over a period of a month as follows: four received 1 drachm daily; four received 2 drachms daily; and four received 3 drachms daily in divided doses for three consecutive weeks. There was no modification of appetite, digestion, bowel action, or of the consistency of the stools. There were no objective signs of disturbance, and no malaise or other subjective symptoms of ill-health.

\section{Summary}

1. Hydrated magnesium trisilicate, as prepared for these experiments, is completely innocuous when taken by mouth even in relatively enormous doses, and does not disturb the normal action of the bowel.
2. In the stomach it exerts a prolonged neutralizing action, which continues for several hours. Its general adsorbent action is similarly continuous. Hydrated silica is produced by its interaction with the gastric juice, and is itself a powerful adsorbent for many substances, including shell-fish poison and pepsin. The pepsin so adsorbed is still available for the purposes of digestion.

3 .'In the small intestine the contents are subjected to the combined adsorptive action of hydrated silica and a proportion of unsplit magnesium trisilicate. The adsorptive affinities of the latter cannot be exhausted quickly, and should therefore be available throughout the whole of the intestinal tract.

4. In the faeces a rise in the percentage of silica occurs.

\section{ALUM-PRECIPITATED TOXOID IN DIPHTHERIA IMMUNIZATION BY}

\section{GEORGE CHESNEY, M.B., D.P.H.}

DEPUTY MEDICAL OFFICER OF HEALTH, POOLE

The procedure at present recommended by most authorities for active immunization with A.P.T. against diphtheria is to give a single dose of $0.5 \mathrm{c.cm}$. or $1 \mathrm{c.cm}$. to children under 8 years (as up to that age reactions are rare), and in children over 8 years to use the Moloney test as a detector of hypersensitive individuals, giving the single dose to Moloney-negative subjects and employing T.A.F. in three doses in those individuals who are Moloney-positive.

\section{Two-shot Immunization with A.P.T.}

The published results of this one-shot method indicate that a fairly high Schick conversion rate can be obtained by the use of a single dose of 1 c.cm., but the rate obtained is not high enough to warrant the omission of a Schick test after immunization to detect those children who have failed to develop immunity. Further, the work of Volk ${ }^{1}$ suggests that the Schick reversion rate may be too high to be ignored: in a group of 170 children who were Schick-negative four to six weeks after receiving one injection of A.P.T. on retesting after six months 9.5 per cent. were found to have reverted to the Schickpositive state. An additional disadvantage in so-called one-shot immunization is the advisability of the use of the Moloney test in children over 8 years. This in actual practice amounts to a two-shot procedure. It is suggested that these disadvantages may be eliminated and routine immunization procedure considerably simplified by using A.P.T. in two small doses. About a year ago, in a private communication, Dr. R. A. O'Brien indicated that in laboratory experiments by Mr. A. T. Glenny A.P.T. in two small doses of 0.1 c.cm., at a three-weeks interval had given a higher and more rapid immunity than a single dose of 0.1 c.cm., and suggested that in children one would expect a similar result.

In $1934 \mathrm{I}^{2}$ found that in children aged 8 to 14 years formol toxoid could be safely employed without the inconvenience of a preliminary Moloney test if an initial dose of $0.1 \mathrm{c} . \mathrm{cm}$. of the toxoid were given subcutaneously, this small dose serving both as a primary antigenic stimulus and as a "detector " of hypersensitive children, the use of the formol toxoid being discontinued and toxoidantitoxin floccules being substituted in the few cases which showed any material degree of reaction to the initial $(0.1$ c.cm.) dose of toxoid. Similarly, in using a small initial dose of A.P.T. one would get warning of hypersensitiveness without the risk of the marked reaction and induration which in sensitive persons results from a dose of $1 \mathrm{c.cm}$., the initial dose of $0.1 \mathrm{c} . \mathrm{cm}$. acting as a detector dose and obviating the use of the Moloney test. 


\section{Practical Results}

On Dr. O'Brien's suggestion 185 children were given by subcutaneous injection two small doses-mostly 0.1 or $0.2 \mathrm{c} . \mathrm{cm}$. and $0.4 \mathrm{c.cm}$. - of A.P.T. at an interval of three to four weeks. Reactions to the initial dose of 0.1 or 0.2 c.cm. were infrequent, and in only one case in which a local reaction $6 \mathrm{~cm}$. in diameter developed was T.A.F. substituted for A.P.T. in the second dose. In fifteen cases (approximately 1 in 12) there was noteworthy local reaction to the second dose, eight of them showing an area of redness of $5 \mathrm{~cm}$. diameter. These reactions to the second dose could have been avoided by a more strict attention to the indication afforded by the first dose, but in a number of cases the parents, though specially requested to report the slightest redness following the initial dose, failed to do so, regarding the redness as a normal concomitant of the injection.

The majority of the reactors were over 8 years of age. One child reported with a very small fluctuating swelling. This was opened and a small amount of sterile " pus" removed. It is probable that dissection into the buttock would disclose in every patient in a V.D. clinic given metallic bismuth or mercury a tiny local necrosis, which is absorbed rapidly and causes no discomfort. Tens of thousands of these injections are carried out annually without any trouble to the patients. It is also probable that when A.P.T. is injected a few leucocytes locally die and are rapidly absorbed. In very rare cases-one in several thousand according to the American figures-a small local abscess occurs which disappears rapidly and causes little trouble. The boy referred to above had suffered a blow on the arm, which probably increased the local damage and caused an abscess to point: it cleared up rapidly and gave little trouble to the patient.

The ages of the children ranged from 1 to 14 years, 148 being under 10 years and thirty-seven being 10 years and over. The children under 10 years old were not Schick-tested in the first place, as during the past two years the Schick-positive rate in children of 10 years and over had been found to be $\mathbf{7 2}$ per cent., this indicating in the younger children a higher Schick-positive rate which did not warrant primary Schick-testing. One hundred and sixty-two of the children were Schick-tested after immunization, 149 being tested six to eight weeks after the second injection and thirteen at a longer interval. Only one of these children gave a positive result, the Schick-negative rate being 99.38 per cent. Of the children Schick-tested after immunization thirty-seven were primary Schick-positives aged 10 to 14 years, and these all showed conversion to the Schick-negative state. It was possible to test the majority of the children with fourfold Schick toxin, this test being regarded as a more stringent indicator of immunity. All the children so tested were negative, indicating a higher average content of circulating antitoxin than is revealed by the ordinary Schick reagents.

A sufficient interval of time has not yet elapsed to permit of any investigation of the Schick reversion rate of the children who have proved negative within two months of their second immunizing dose, but it seems reasonable to assume that the reversion rate will be no higher, and very possibly lower, than that found following a single dose.

\section{Summary}

One hundred and eighty-five children aged 1 to 14 years were treated with two small doses of A.P.T. at an average interval of four weeks. Moloney testing was not practised, as the small initial dose gave a safe and adequate warning of probable sharp reactors. A 99.38 per cent. Schick-negative rate was obtained in 162 children Schicktested six or more weeks after the second dose. It is suggested that this two-shot procedure with A.P.T. is a simple and efficient method of immunization against diphtheria. Reaction difficulties are minimized without the use of the inconvenient Moloney test, and the high Schick-negative rate obtained, if consistently maintained, indicates the possible further simplification of procedure by the justifiable omission of the post-Schick test.

REFEREITES

1 Volk, V. K.: Amer. Journ. Pub. Health, 1935, xxv, 430.

Chesney, G.: Med. Officer, November 2nd, 1935, p. 186.

\section{IMMUNIZATION AGAINST DIPHTHERIA WITH ALUM-PRECIPITATED TOXOID (A.P.T.) \\ BY}

H. J. PARISH, M.D., M.R.C.P.ED.

(Wellcome Physiological Research Laboratories, Beckenham, Kent)

It has recently been suggested in the British Medical Journal (November 9th, 1935, p. 908) that the success of " one-shot" immunization against diphtheria is largely dependent on the basal immunity of a community. Where the natural Schick-negative rate is high a single injection of 0.5 c.cm. or $1 \mathrm{c} . \mathrm{cm}$. of alum-precipitated toxoid (A.P.T.) will convert well above 90 per cent. of Schickpositive individuals to Schick-negative within a few weeks, but where the Schick-negative rate is low the conversion figure may be much lower. Unless one can control the " one-shot" method by post-inoculation Schick-testing a course of two doses-namely, $0.1 \mathrm{c.cm}$. followed by $0.5 \mathrm{c} . \mathrm{cm}$. two to four weeks later-should be substituted, as it has been shown on animals as well as on small groups of children that two doses give better immunity.

We have recently compared the " one-shot" and twodose methods in a residential school and in a nursery school in which the natural immunity rates were low.

\section{Results in a Residential and a Nursery School}

In the residential school, on the outskirts of London, the natural Schick-negative rate was only 20 per cent., which was unexpected, as about two-thirds of the children were from London or towns in the provinces, and must have had many opportunities of contact with the diphtheria bacillus. The ages of the children ranged from 7 to 16 years, the majority being under 12 years. Immunization was carried out with batches of A.P.T. which had proved highly effective elsewhere, but only eightyfive of 165 children (51 per cent.) were Schick-negative within five weeks of a single injection of 0.5 or $1 \mathrm{c.cm}$. of prophylactic, and only 206 of 321 children (64 per cent.) within fifteen weeks of the injection. The two-dose method (in this instance $0.1 \mathrm{c.cm}$. followed by only 0.2 c.cm. three weeks later) converted to Schick-negative thirty-one of thirty-five children (89 per cent.) within five weeks of the commencement of immunization, and all thirty-five (100 per cent.) within fifteen weeks, a much more satisfactory result. A few of the Schick-positive reactors among the older children had been Moloneytested and thirteen had given weak positive reactions. Contrary to our ordinary practice, which is to employ T.A.F. for Moloney-positive subjects, it was decided to immunize these children also with A.P.T., 0.1 c.cm. followed by $0.2 \mathrm{c} . \mathrm{cm}$. This procedure was justified by the results with the particular batches of prophylactic we were using, as only a few mild or moderate local reactions were encountered. As a purely precautionary measure games were discontinued for one day only for children 